

\section{Analysis of Interfacial Effects in}

\section{All-Solid-State Batteries with Thiophosphate Solid Electrolytes}

Anton Neumann, ${ }^{*, \dagger, \ddagger}$ Simon Randau, ${ }^{\boldsymbol{\top}, \S}$ Katharina Becker-Steinberger, ${ }^{\dagger, \ddagger}$ Timo Danner, ${ }^{\dagger, \ddagger}$ Simon Hein, ${ }^{\dagger, \ddagger}$ Ziyang Ning, ${ }^{\prime}$ James Marrow, $\|$ Felix H. Richter,, , Jürgen Janek, $, \S, \perp$ and Arnulf Latz ${ }^{\dagger, \ddagger, \# ~}$

$\dagger$ †erman Aerospace Center (DLR), Institute of Engineering Thermodynamics, Pfaffenwaldring 38-40,70569 Stuttgart, Germany

$\ddagger$ Helmholtz Institute for Electrochemical Energy Storage (HIU), Helmholtzstraße 11, 89081 Ulm, Germany

【Institute of Physical Chemistry, Justus-Liebig-University Giessen, Heinrich-Buff-Ring 17, 35392 Giessen, Germany.

$\S$ Center for Materials Research (LaMa), Justus-Liebig-University Giessen, Heinrich-Buff-Ring 16, 35392 Giessen, Germany

||Department of Materials, University of Oxford, Parks Road, Oxford OX1 3PH, United Kingdom

$\perp$ Battery and Electrochemistry Laboratory (BELLA), Institute of Nanotechnology, Karlsruhe Institute of Technology, Hermann-von-Helmholtz Platz 1, 76344 Eggenstein-Leopoldshafen, Germany \#Ulm University (UUlm), Institute of Electrochemistry, Albert-Einstein-Allee 47, 89081 Ulm, Germany

E-mail: anton.neumann@dlr.de

Abstract 
All-solid-state batteries (ASSBs) present a promising route towards safe and high power battery systems in order to meet the future demands in the consumer and automotive market. Composite cathodes are one way to boost the energy density of ASSBs compared to thin-film configurations. In this manuscript, we investigate composites consisting of $\beta-\mathrm{Li}_{3} \mathrm{PS}_{4}$ ( $\beta$-LPS) solid electrolyte and high energy $\mathrm{Li}\left(\mathrm{Ni}_{0.6} \mathrm{Mn}_{0.2} \mathrm{Co}_{0.2}\right) \mathrm{O}_{2}$ (NMC622). The fabricated cells show a good cycle life with a satisfactory capacity retention. Still, the cathode utilization is below the values reported in the literature for systems with liquid electrolytes. The common understanding is that interface processes between the active material and solid electrolyte are responsible for the reduced performance. In order to throw some light on this topic, we perform 3D microstructureresolved simulations on virtual samples obtained via X-ray tomography. Through this approach, we are able to correlate the composite microstructure with electrode performance and impedance. We identify the low electronic conductivity in the fully lithiated NMC622 as material inherent restriction preventing high cathode utilization. Moreover, we find that geometrical properties and morphological changes of the microstructure interact with the internal and external interfaces, significantly affecting the capacity retention at higher currents.

\section{Keywords}

all-solid-state batteries, thiophosphate solid electrolyte, 3D microstructure-resolved simulations, nickel-rich layered oxides, impedance analysis, micro-computer tomography

\section{Introduction}

In order to promote the success of electric vehicles by increasing their driving range, battery research needs to surpass the performance limitations of lithium-ion batteries with liquid electrolytes. ${ }^{?}$ All-solid-state batteries (ASSB) present a promising route towards a high energy and power storage system. The key advantages of ASSBs result from the solid electrolyte, 
serving simultaneously as a separator and providing ionic paths in the positive electrode composite. Furthermore, it may enable the use of Li-metal as anode material, which is inevitable to meet the energy density of lithium-ion batteries with liquid electrolyte. ? One promising type of ASSB uses thiophosphate solid electrolytes. For example, $\beta-\mathrm{Li}_{3} \mathrm{PS}_{4}$ ( $\beta$-LPS) forms a suitable interface with lithium metal and can be used in a broad voltage range with various active materials like nickel rich layered oxides (NMC) or classical lithium cobalt oxide (LCO), ? ? once these are coated with films protecting the electrolyte against oxidation. An important advantage of lithium thiophosphates is that they can be processed at ambient temperature into highly conducting and dense sheets or pellets. Thus, the preparation of composite cathodes with good interfacial contact is facilitated. ? This leads to a low charge transfer resistance and improved cycling performance.

However, despite their many advantages, the current rate performance of state-of-the-art thiophosphate cells does not meet expectations. A fundamental understanding of the dissipative processes that cause these performance losses is indispensable. At the same time, the need for performance prediction and investigation of cell architectures and designs is increasing due to the rapidly growing number of material combinations and cell geometries. In order to investigate both, complementary theoretical and experimental tools are of particular importance. Among the theoretical approaches, computer-aided continuum simulations are particularly well suited for such design studies.

Most existing solid electrolyte and ASSB models focus on film-type designs. ? ? ? ? ? ? ? ? The main emphasis in these studies is to investigate the coupling of local charge imbalances, electric fields, and electromechanical forces and their effect on the charge transfer reaction at the interface. In particular, there is an increasing interest in mechanical challenges at the interface, such as delamination, ${ }^{?}$ mechanical stresses induced by lithium plating and dendrite formation, ${ }^{?}$ or the influence of the coupling of Maxwell stresses and elastic stresses to transport.?

Recently, there has been a tremendous progress in the imaging of ASSBs through computer 
tomography, enabling in-situ studies on working cells and providing valuable inside on degradation phenomena and secondary phase formation. ? ? ? We refer to Pietsch and Wood? for a general overview of imaging techniques for battery materials. Also oxide-based ASSBs with composite positive electrodes are studied by means of 3D microstructure-resolved simulations. ? ? ? For example, Finsterbusch et al. ${ }^{?}$ used a 3D reconstruction of the manufactured oxide-based composite electrode as input for microstructure-resolved continuum simulations for the first time.

In this work, we present a combination of theory and experiments to understand the limiting factors for the electrochemical performance of $\beta$-LPS based ASSBs. The systems under investigation consist of a composite cathode containing $\beta-\mathrm{Li}_{3} \mathrm{PS}_{4}(\beta$-LPS) and $\mathrm{Li}\left(\mathrm{Ni}_{0.6} \mathrm{Mn}_{0.2} \mathrm{Co}_{0.2} \mathrm{O}_{2}\right)(\mathrm{NMC622})$, a $\beta$-LPS separator, and a lithium metal anode. The cells are imaged via micro-computer tomography (CT) to analyze the microstructural properties of all-solid-state batteries and provide virtual electrode reconstructions used as the computational input.

In the simulation part of this study, we examine the interaction between electrode microstructure and the intrinsic material properties on the electrochemical performance under discharge. To account for changes in material properties during cycling, the 3D microstructureresolved modeling framework is extended by incorporating state-of-charge (SOC) dependent transport parameters. This approach enables us to investigate qualitatively the influence of various physical parameters, such as ionic and electronic conductivity, as well as microstructural effects, such as inhomogeneous composition and delamination, on the overall cell performance.

The simulations are validated against experiments and we can report qualitative agreement without any modification or fitting of the parameters. The microstructure-resolved simulations enable us to identify and locate bottlenecks in the system and provide a time and cost-efficient tool for sensitivity analysis of multiple input parameters. The combination of computational and experimental techniques allows deducing design guidelines for cell opti- 
mization, which is the basis for a knowledge-based development of future ASSBs.

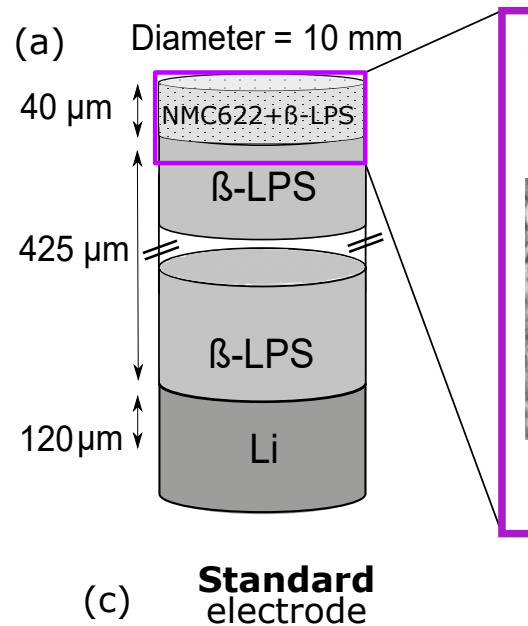

(1)

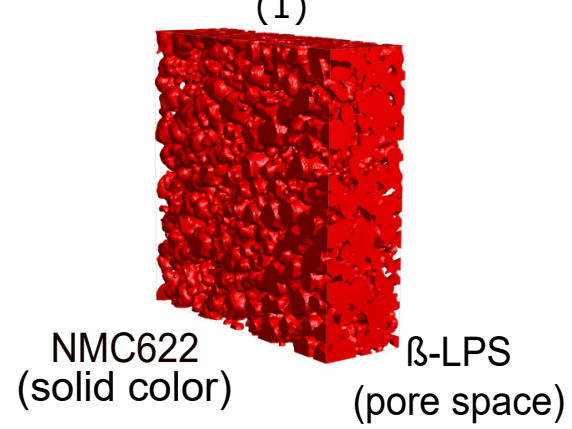

(b) Computer tomography
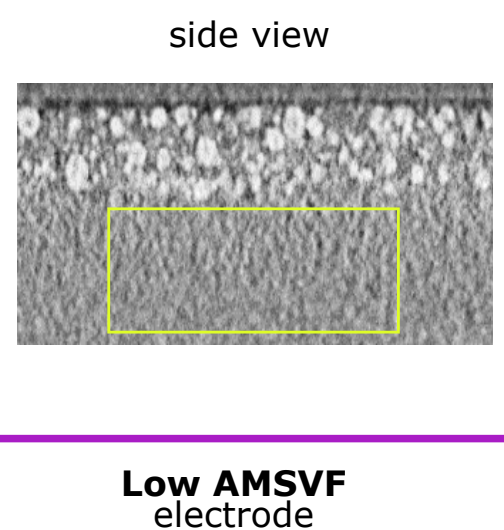

(2)

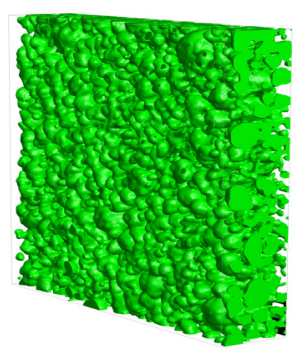

top view

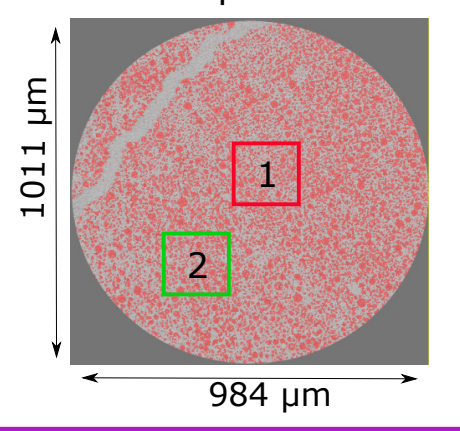

High energy

Figure 1: (a) Schematic of the cell setup and corresponding layer thicknesses. (b) CT images inside ( $x y$-plane) and top view (yz-plane) showing the pristine composite cathode and parts of the compressed separator (yellow inset). (c) 3D microstructure reconstruction of the active material distribution for the region 1 (red) and region 2 (green). $\beta$-LPS is transparent in this representation. The blue structure represents the high energy setup with a thickness twice as the standard cell.

\section{Experiment}

\subsection{Electrode and cell preparation}

Materials Thiophosphate solid electrolyte $\beta-\mathrm{Li}_{3} \mathrm{PS}_{4}\left(1.2 \cdot 10^{-4} \mathrm{~S} / \mathrm{cm}\right)$ and uncoated active material $\mathrm{Li}\left(\mathrm{Ni}_{0.6} \mathrm{Mn}_{0.2} \mathrm{Co}_{0.2}\right) \mathrm{O}_{2}$ (NMC622) for this study were provided by BASF SE. The chemicals were stored in a glovebox filled with argon. NMC622 active material was dried in a Büchi furnace at $250^{\circ} \mathrm{C}$ over night. The secondary particle size of the active material was 
around $10 \mu \mathrm{m}$. A $120 \mu \mathrm{m}$ thick lithium foil disc with $9 \mathrm{~mm}$ diameter was used as anode.

Cathode composite The composite consisted only of dried NMC622 and $\beta$-LPS. The mass ratio was 70:30 (volume ratio 47:53) of NMC622 and $\beta$-LPS, respectively. The powders were mixed in an agate mortar by hand for $15 \mathrm{~min}$. The composite mixture was prepared on the same day before cell assembly. In order to investigate the essential cell kinetics and performance of the composite cathode, we did not use any additives like binder or carbon black.

Assembly of Li| $\beta$-LPS|NMC cells All cell tests were carried out using a cell casing manufactured in house. ? For assembly, one stainless steel stamp was used for closing the polyether ether ketone (PEEK) cylinder. The cylinder had a diameter of $10 \mathrm{~mm}$. All materials are added via the remaining opening. $60 \mathrm{mg}$ of the $\beta$-LiPS solid electrolyte was added first. This powder was uniformly compressed by hand. Afterwards $10 \mathrm{mg}$ of cathode composite was added and distributed evenly. This amount corresponds to an active material loading of $8.9 \mathrm{mg} / \mathrm{cm}^{2}$ and $1.78 \mathrm{mAh} / \mathrm{cm}^{2}\left(Q_{\text {theo }}=200 \mathrm{mAh} / \mathrm{g}\right)$. This powder stack was then compressed uniaxially at $30 \mathrm{kN}$ (approx. $380 \mathrm{MPa}$ ) for three minutes. After compacting the pellet, the lithium metal anode was added. The lithium foil was placed on the $\beta$-LPS solid electrolyte and a copper foil was added on the lithium towards the stainless steel current collector. An external frame was used around the cell casing during all the following tests, applying a constant pressure of approximately $50 \mathrm{MPa}$. The schematic cell setup is shown in Fig. ??.

For the high energy all-solid-state batteries the amount of the separating solid electrolyte was halved to $30 \mathrm{mg}$ and the amount of cathode composite was doubled to $20 \mathrm{mg}$. This corresponds to $17.8 \mathrm{mg} / \mathrm{cm}^{2}$ and $3.56 \mathrm{mAh} / \mathrm{cm}^{2}$. Furthermore, the applied pressure to compact the powders was only $25 \mathrm{kN}$ for three minutes in order to avoid crack formation. 


\section{$2.2 \quad$ Electrochemical characterization}

Charge and discharge tests were performed using a VMP-300 Biologic or MACCOR potentiostat/galvanostat. Cells with lithium metal anode were charged to $4.3 \mathrm{~V}$ and discharged to $2.6 \mathrm{~V}$ versus $\mathrm{Li}^{+} / \mathrm{Li}$ at $25^{\circ} \mathrm{C}$. The $\mathrm{C}$-rate for charge and discharge experiments was $0.1 \mathrm{C}$, corresponding to $0.178 \mathrm{mAh} / \mathrm{cm}^{2}$ for cells with $8.9 \mathrm{mg} / \mathrm{cm}^{2}\left(1.78 \mathrm{mAh} / \mathrm{cm}^{2}\right)$ active material loading. With a higher active material loading and for rate tests the current densities were in-

creased accordingly. The electrochemical impedance spectroscopy (EIS) measurements were carried out with a VMP-300 potentiostat. Before the EIS step was performed, 10 minutes open circuit potential was measured for relaxation of the test cells. The EIS measurement was conducted in a frequency range of $7 \mathrm{MHz}$ to $1 \mathrm{~Hz}$, applying a $10 \mathrm{mV}$ amplitude.

\subsection{Computer Tomography}

In order to reduce the workload for image acquisition, we focus the imaging activities on samples with a material loading of $8.9 \mathrm{mg} / \mathrm{cm}^{2}$. The X-ray computed tomography was taken with Xradia Versa 510 X-ray Microscope, operated at $80 \mathrm{kV}$ to image with a voxel size of $0.967 \mu \mathrm{m}$ in a field of view of $977.66 \times 977.66 \times 947.66 \mathrm{\mu m}^{3}$. The general computer tomography measurement parameters and structural parameters are listed in Tab. S1 of the supporting information.

\section{Simulation}

\subsection{Simulation Framework}

The simulation results in this study are generated within the Battery and Electrochemistry

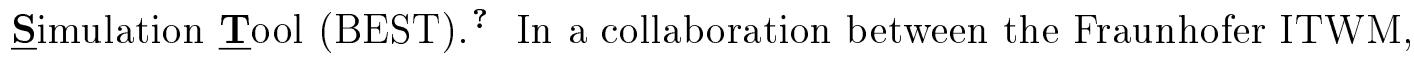
Kaiserslautern and the DLR/HIU the original framework for lithium-ion batteries with liq- 
uid electrolytes is constantly improved and extended to describe additional effects as well as new battery chemistries and concepts. The dynamic evolution of the concentration and the potential distribution in the simulated cell system is described by the conservation equations for mass, charge, and energy. ? ? The consistent derivation of this coupled set of partial differential equations is based on the fundamental principles of non-equilibrium thermodynamics.

A prominent feature of our simulation framework is the ability to perform microstructureresolved simulations on virtual electrodes consisting of a voxel-based 3D image. ? $\mathrm{Mi}^{-}$ crostructural effects, like the particle shape or the tortuosity of the electrodes, are intrinsically included. A short overview of our model equations and the simulation methodology for the calculation of impedance spectra is provided in. ? For a more rigorous derivation of the thermodynamically consistent battery model we refer to the work of Latz et al. ? ? Since our 3D microstructure simulation resolution is typically larger than the width of charge imbalances, so-called space-charge layers (SCL), in the vicinity of interfaces, we neglect the influences of SCLs in our simulation. A detailed analysis of lithium ion transport phenomena and interfacial processes including SCLs on a nanometer scale within inorganic solid electrolytes can be found in? ?

\subsection{Model Parametrization}

In this study we extract transport, kinetic, and thermodynamic parameters from different sources in the literature, as well as our own experiments. This data sets the ground for a sound parametrization of the $\beta$-LPS electrolyte as well as the NMC622 cathode active material.

Bulk Transport In order to capture the single ion conductor characteristics of $\beta$-LPS, ? ? the transference number of lithium ions is set to $t_{\mathrm{Li}}^{+}=1$. Several articles report bulk transport properties of $\beta$-LPS electrolyte. ? ? The lithium ion diffusivity $\left(D_{\mathrm{Li}}^{\mathrm{elyt}}=2.4 \cdot 10^{-9} \mathrm{~cm}^{2} / \mathrm{s}\right)$ in $\beta$ - 
LPS at room temperature $\left(25^{\circ} \mathrm{C}\right)$ is based on Nuclear Magnetic Resonance measurements. Values for the ionic conductivities reported in the literature range from $1.6 \mathrm{mS} / \mathrm{cm}^{?}$ ? to $1.09 \mathrm{mS} / \mathrm{cm}^{?}$ depending on the measurement technique. We select the ionic conductivity value of $\sigma_{\mathrm{Li}}^{\text {elyt }}=1.20 \cdot 10^{-4} \mathrm{~S} / \mathrm{cm}$ based on the electrochemical impedance spectroscopy measurement presented in a previous work. ${ }^{?}$ The initial Li-ion concentration in the solid electrolyte $c_{\mathrm{Li}}^{\text {elyte, } 0}=0.0103 \mathrm{~mol} / \mathrm{cm}^{3}$ is calculated via the density and composition of $\beta$-LPS. We calculate the non-ideality factor $f_{\mathrm{Li}}^{\text {elyte }}=1.28$ for cation site limited transport in bulk $\beta$-LPS based on the work by Braun et al. ? The transport parameters for NMC622 are the SOC dependent electronic conductivity and the chemical diffusion coefficient of lithium. The work by Noh et al., ${ }^{\text {? }}$ Amin et al. ${ }^{?}$ and Zhou et al. ${ }^{?}$ provide experimental measurements for these parameters. Since they use a liquid electrolyte, these results serve us as an estimate for our simulations with the thiophosphate solid electrolyte. Particularly, the electronic conductivity of NMC622 shows a significant decrease by up to four orders of magnitude during lithiation from $\sigma_{\mathrm{Li}=0.4}^{\mathrm{NMC}}=2 \cdot 10^{-2} \mathrm{~S} / \mathrm{cm}$ down to $\sigma_{\mathrm{Li}=1}^{\mathrm{NMC}}=1.6 \cdot 10^{-6} \mathrm{~S} / \mathrm{cm}$. The SOC dependence of the chemical Li diffusion coefficient is less pronounced compared to the conductivity, but still varies in the range of $D_{\mathrm{Li}=0.4}^{\mathrm{NMC}}=1.83 \cdot 10^{-11} \mathrm{~cm}^{2} / \mathrm{s}$ to $D_{\mathrm{Li}=1}^{\mathrm{NMC}}=3.10 \cdot 10^{-12} \mathrm{~cm}^{2} / \mathrm{s}$.

Electrochemical reactions The characterization of bulk transport parameters is relatively straightforward compared to the parametrization of interface reactions. Above all, the formation of side products and the loss of contact area due to swelling and shrinking of the active material introduces significant uncertainties in the kinetic parameters. Therefore, we extract parameters from different experiments in the pristine state. First, kinetic parameters of the Li charge transfer at the anode are determined from impedance data measured on symmetrical lithium cells with a $\beta$-LPS separator. The corresponding data is given in Fig. S3 of the supporting information. In the next step, we focus on the reactions at the composite cathode. The deconvolution of the cathode interface reactions based on half-cell measurements is not as straightforward as in the symmetrical Li cells. Therefore, we man- 
ually tune the initial charge transfer rate and corresponding differential capacities in order to reproduce half-cell measurements as accurately as possible. The kinetic parameters of the two half-cell reactions e.g. the exchange current density are estimated based on the experimental impedance data (cf. supporting information). Our analysis indicates that the anodic interface reaction can be seen at slightly higher frequencies compared to the intercalation reaction at the cathode. Since we parameterize the cathode interface reaction based on the impedance measurements after the first cycle, effects like the initial solid electrolyte decomposition are inherently included.

All thermodynamic parameters, especially, the open circuit potential (OCP) of the NMC622, are measured with respect to a lithium metal electrode. Therefore, we set the OCP of the $\mathrm{Li}$ metal electrode in our simulations as a reference $U_{0}^{\mathrm{Li}}=0 \mathrm{~V}$. The OCP of NMC622 is given as a function of the SOC in the supporting information (Eq. S 4.3). All simulation parameters are also summarized in Tab. ??.

\subsection{Simulation domain}

In analogy to the experimental setup, we use in our simulations virtual cells consisting of a composite cathode, a $\beta$-LPS separator and a lithium metal anode. The lithium metal and the separator are modeled as isotropic layers with the corresponding separator thickness, depending on the cell setup. In our simulations, we investigate the influence of structural inhomogeneities and modifications on cell performance and impedance. A description of the preparation of the virtual composite cathodes is given in the next paragraphs. All virtual samples are analyzed regarding their specific surface area for lithium intercalation, as well as their effective transport and structural properties in the solid electrolyte and active material using the commercial software GeoDict. ? The results of the structural analysis and corresponding electrochemical simulations are provided in Sec. ?? and Sec. ??, respectively. 
Table 1: Simulation Parameters in BEST. Values are taken from the respective reference (superscript [\# of REF]), measured by the authors (superscript $\left[^{\circ}\right]$ ), or calculated (superscript $\left[{ }^{*}\right]$ ). The chemical diffusion coefficient and the electronic conductivity of NMC622 are functional parameters dependent on the SOC of the material. Their values are given for the initial concentrations. All other parameters are constant during the simulations.

\begin{tabular}{|c|c|c|}
\hline parameter, unit & description & value \\
\hline \multicolumn{3}{|l|}{ Electrolyte } \\
\hline$c_{\mathrm{I}}^{\text {elyte }, 0}, \mathrm{~mol} / \mathrm{cm}^{3}$ & concentration of Li ions in $\beta$-LPS ${ }^{*}$ & 0.0103 \\
\hline$\kappa^{\text {elyte, }, 0}, \mathrm{~S} / \mathrm{cm}$ & Li-ion conductivity ${ }^{\circ}$ ? & $1.20 \cdot 10^{-4}$ \\
\hline$t_{\mathrm{Li}}^{+}$ & transference number of Li ions * & 1 \\
\hline$D_{\mathrm{Li}}^{\text {elyte }}, \mathrm{cm}^{2} / \mathrm{s}$ & Li-ion self diffusion coefficient? & $2.4 \cdot 10^{-9}$ \\
\hline$f_{\mathrm{Li}}^{\text {elyte }}$ & thermodynamic correction factor* & 1.28 \\
\hline$\beta$-LPS, $\mu \mathrm{m}$ & electrolyte (separator) thickness ${ }^{\circ}$ & 425 \\
\hline \multicolumn{3}{|l|}{ NMC622 } \\
\hline$c_{\mathrm{Li}}^{\mathrm{NMC}, 0}, \mathrm{~mol} / \mathrm{cm}^{3}$ & initial Li concentration * & 0.021 \\
\hline$\sigma_{\mathrm{Ii}}^{\mathrm{NMC}}, \mathrm{S} / \mathrm{cm}$ & electronic conductivity? & Eq. S 4.1 \\
\hline$D_{\mathrm{Li}}^{\mathrm{NMC}}, \mathrm{cm}^{2} / \mathrm{s}$ & Li-ion chemical diffusion coefficient? & Eq. S 4.2 \\
\hline$U_{0}^{\mathrm{NMC}}, \mathrm{V}$ & open circuit potential? & Eq. S 4.3 \\
\hline$\alpha^{\mathrm{NMC}}$ & symmetry factor & \\
\hline$c_{\mathrm{Li}}^{\mathrm{NMC}, \max }, \mathrm{mol} / \mathrm{cm}^{3}$ & maximum Li concentration * & 0.0519 \\
\hline$i_{00}^{\mathrm{NMC}}, \mathrm{A} / \mathrm{cm}^{2} / \mathrm{mol}^{1.5}$ & exchange current density factor * & $4.98 \cdot 10^{-4}$ \\
\hline$C_{\mathrm{DL}}^{\mathrm{NMC}}, \mathrm{F} / \mathrm{cm}^{2}$ & double layer capacity * & $8 \cdot 10^{-7}$ \\
\hline$L_{\mathrm{NMC}}, \mu \mathrm{m}$ & composite cathode thickness ${ }^{\circ}$ & 40 \\
\hline \multicolumn{3}{|l|}{ Li Metal } \\
\hline$\sigma_{\mathrm{Ij}}^{\text {elode }}, \mathrm{S} / \mathrm{cm}$ & electronic conductivity * & $10 \cdot 10^{2}$ \\
\hline$U_{0}^{\mathrm{Li}}, \mathrm{V}$ & open circuit potential & 0 \\
\hline$\alpha^{\mathrm{Li}}$ & symmetry factor & 0.5 \\
\hline$i_{00}^{\mathrm{Li}}, \mathrm{A} / \mathrm{cm}^{2}$ & exchange current density factor * & $8.87 \cdot 10^{-4}$ \\
\hline$C_{\mathrm{DI}}^{\mathrm{Li}}, \mathrm{F} / \mathrm{cm}^{2}$ & double layer capacity * & $2 \cdot 10^{-7}$ \\
\hline
\end{tabular}




\subsubsection{Electrode reconstruction}

The basis for all virtual cathode samples in this section are the reconstructions resulting from the CT measurements described in Sec. ??. The grayscale images are binarized in GeoDict? via a manual threshold and converted into a voxel based geometry. The total reconstructed composite cathode $\left(1011 \times 984 \times 50 \mathrm{~mm}^{3}\right)$ with a resolution of $1 \mu \mathrm{m}$ cropped from the tomographed volume is shown in Fig. ??(b). Based on the electrode composition and density of the materials, the theoretical active material solid volume fraction (AMSVF) of the cathode is in the range of $46 \mathrm{vol}-\%$. The manual threshold in the binarization step is set accordingly to get the same AMSVF in the virtual sample. Finally, a representative volume of the fully reconstructed cathode is cropped to the desired virtual electrode size of $150 \times 150 \times 40 \mathrm{\mu m}^{3}$ to be processed in BEST. The reduced virtual electrode volume is determined based on a trade-off between the simulation accuracy and running time.

Visual inspection of tomography data reconstruction reveals fluctuations in the in-plane composition of the fully reconstructed cathode. Depending on the choice of the selection area the AMSFV is 46 vol-\% in Fig. ??(red square) and 38 vol-\%(green square), respectively. In order to evaluate the influence of the varying material fraction on the performance, we create virtual electrodes for each selected area, which are shown in Fig. ??(c). The reconstruction areas are selected to have no overlap, in order to provide individual structures without resemblance. Additionally, the virtual electrodes are trimmed by a few voxels at the interface to the current collector to provide a sufficient electrical contact. The influence of this processing step will be discussed in detail below. In the remainder of this article, these two samples will be referred to as "standard" and "low AMSVF" virtual electrode, respectively.

Since we do not perform CT measurements on the high energy electrodes described in Sec. ??, the microstructure of the corresponding virtual sample is generated by duplicating the standard electrode with 46 vol- $\%$ active material to reproduce the electrode thickness of $80 \mu \mathrm{m}$. 


\subsubsection{Microstructure Modification}

In our electrochemical simulation, we do not track the evolution of the electrode microstructure due to mechano-chemical processes. Still, we are able to investigate the effect of structural changes that might occur due to mechanical stress and interface degradation in our simulations by virtually introducing microstructural modifications. This approach allows us to link possible structural changes, as well as changes in material properties, to characteristic features in the corresponding electrochemical measurements. In this article, we will focus on two different mechanical processes affecting interfaces in all-solid-state batteries. (I) The increasing contact area at the interface of the composite cathode with the current collector (external interface) and (II) delamination of the active material particles from the solid electrolyte network (internal interface).

Cathode to Current Collector Interface It is known that the particle distribution and size at the interface to the current collector is pivotal for the overall cell performance of ASSBs. ? ? ? Indications for an unfavorable distribution in the reconstructed samples are discussed in Sec. ??. We perform a parameter study for different contact areas between cathode active material and current collector to quantify the effect of the contact resistance at this external interface. This change in direct contact area of the cathode $\left(A_{\mathrm{C}}\right)$ is introduced by cropping (cf. Fig. ??(a)) the standard sample voxel by voxel at the interface to the current collector. Through this procedure, the increasingly larger AMSVF improves the contact to the current collector. The qualitative influence of the contact loss on the cell performance will be investigated in detail in Sec. ??.

Particle Delamination Through the CT reconstruction, we obtain the material distribution. This approach gives us a first approximation of the experimental microstructure. Nevertheless, features like a contact loss of individual particles, reduced electrolyte wetting, and remaining void space after processing are in the submicrometer range and, thus, below 
(a)

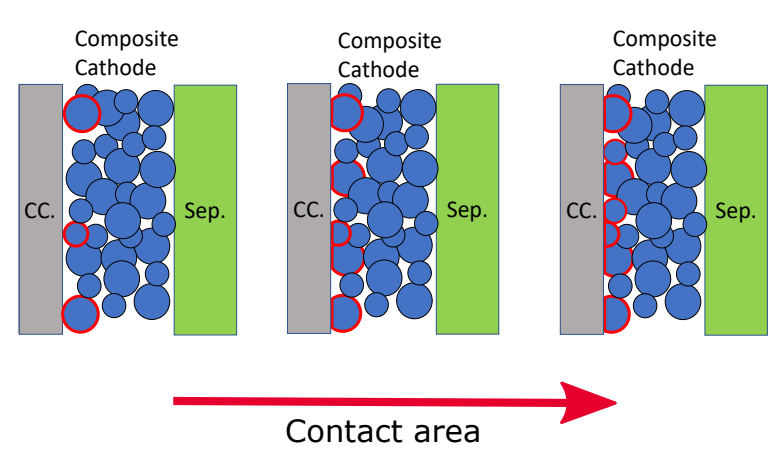

(b)

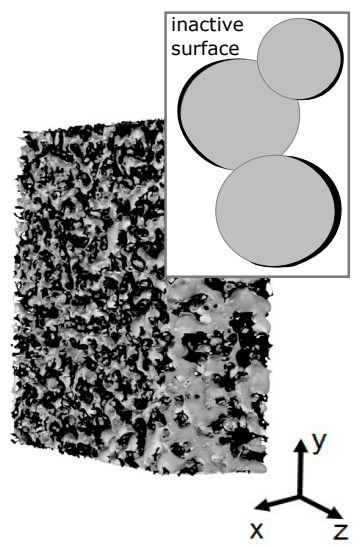

Figure 2: (a) Schematic for the cropping routine to increase the current collector to cathode contact interface $\left(A_{\mathrm{C}}\right)$ in the simulated reconstruction (blue $=$ NMC622; pore space $=$ $\beta$-LPS). (b) Exemplary microstructure generated by the particle delamination procedure. Inactive surface areas for the active material (grey) induced through the delamination are shown in black and the original ionic network is transparent.

the resolution of the X-ray CT measurements.

The work by Koerver et al. ${ }^{\text {? }}$ for nickel rich layered oxides and $\beta$-LPS indicates that a partial delamination of the active material occurs for this type of solid-state system during cycling. The origin of the delamination lies in the anisotropic volume change of NMC622 of up to $5 \%$ during lithiation and delithiation. This particle breathing leads to local stress in the cathode and subsequently to a partial electrochemical deactivation and capacity loss. Unlike polymer-based or even liquid electrolytes, $\beta$-LPS is unable to reoccupy the emerging void space after stress release.

In our geometrical model, we capture the delamination effect after the initial charging step by introducing a passivating coating layer of solid electrolyte on the NMC622 particle surface. We deactivate the lithium transport across the interface of the passivating electrolyte layer and active material, which leads to a reduction of active surface area in the cathode. By controlling the degree of active surface passivation, we are able to mimic different degrees of internal interface delamination. Thereby, the transport across and through the passivating solid electrolyte is kept unchanged and preserves the unmodified solid electrolyte network. The resulting structure modification through delamination is schematically shown in Fig. 
??(b). We assume that the volume change and the induced local stresses lead to a densification in this inter-particle region and prevent full contact loss of the active material. Therefore, the conductive network for lithium ion transport stays intact. A detailed study on the influence of this particle delamination process on cell performance is discussed in Sec. $? ?$.

\section{Results and Discussion}

\subsection{Structural Analysis}

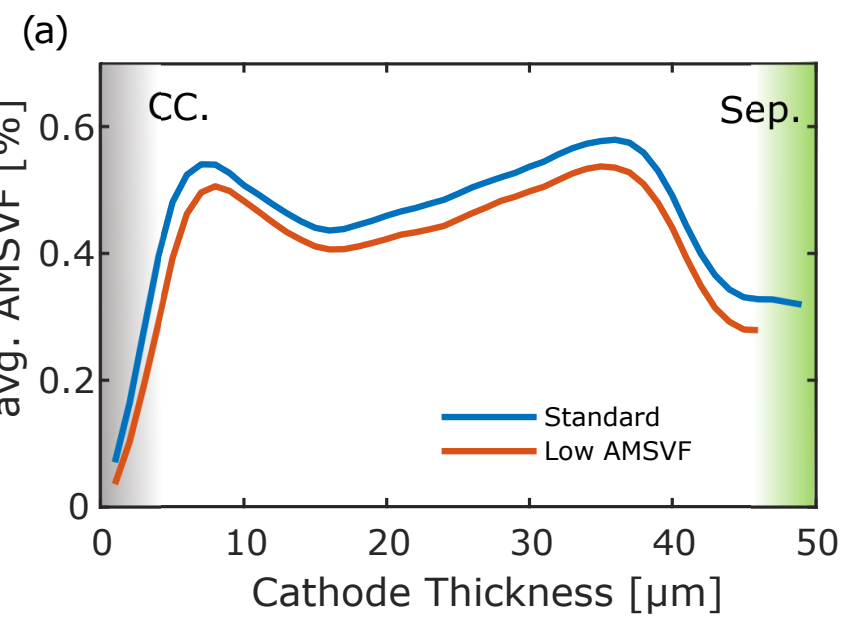

(b1)

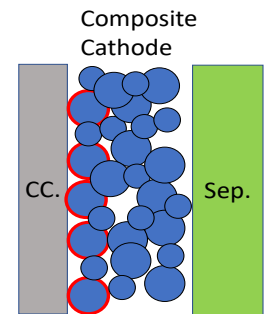

(b2)

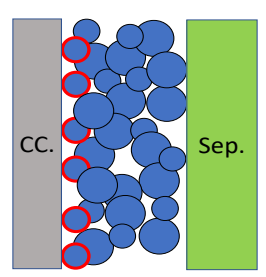

Figure 3: (a) Averaged layer AMSVF for the active material in the x-direction, which indicates particle migration. (b) Current collector to cathode composite interface under (1) protrusion of larger particles towards the current collector and (2) trapping of smaller particles close to the current collector.

The electrochemical performance of ASSBs and Li-ion batteries, in general, is strongly influenced by the structural properties of the electrodes. ? Analyzing the structure regarding material distribution and resulting effective transport parameters, e.g. conductivity, diffusivity, and tortuosity improves the understanding of the battery performance and reveals potential bottlenecks. In this section, we investigate the structural properties of the 
reconstructed samples and structurally modified virtual samples described in Sec. ??.

The three reconstructed virtual electrodes (Fig. ??(c)) differ in their composition, and therefore, their geometric properties are affected. The calculated tortuosity $\tau$ via GeoDict? of the electrolyte phase in the standard, low AMSVF, and high energy virtual electrodes are given in Fig. ??(a). In general, the tortuosity in through-plane direction $\mathrm{x}$ is found to be slightly smaller compared to the values in the in-plane directions y and z. The tortuosity values indicate an improved Li transport due to the lower AMSVF in the low AMSVF sample compared to the standard electrode of $7-10 \%$. Since the high energy cell is generated by duplicating the standard sample (cf. Sec. ??), tortuosity values deviate by less than $1 \%$.

Fig. ??(a) shows the AMSVF distribution in through-plane direction for two different subregions introduced in Sec. ??. Although the total AMSVF between both samples deviates up to $10 \%$, the material distribution shows the same qualitative features. Detailed analysis of the AMSVF in the $\mathrm{x}$-direction for both reconstructed virtual samples reveals two prominent peaks indicating an active material redistribution in the cathode (cf. Fig. ??). One reason for the peak formation could originate from the cathode pressing, where both outer surface layers experience a higher densification than the center part. A similar material compression and porosity reduction can be observed for calendered electrodes in liquid lithium-ion battery systems. ' Another reason could be the migration of particles during the cathode mixing process, ${ }^{?}$ leading to clustering of larger active material particles.

Based on the structural analysis and the $\mathrm{CT}$ reconstruction, we can conclude that larger particles agglomerate close to the current collector. Smaller particles are either trapped close to the current collector or migrate to the electrode surface towards the separator. The change in the cathode interface composition through larger and smaller protruding particles is schematically shown in Fig. ??(b). X-ray CT images illustrating the discussed particle distribution are shown in Fig. S4-S5 of the supporting information. 
Although the discussed processing effects cannot be fully discriminated, they both result in a low AMSVF at the current collector interface, potentially leading to a reduced electronic connectivity of the composite electrode layer. A quantitative analysis of this effect is provided in the paragraph below.

Cathode to Current Collector Interface For the investigation of the cathode contact area reduction to the current collector the electronic conductivity is the decisive property for cell performance. We define the calculated cathode surface area in direct contact to the current collector as $A_{\mathrm{C}}$. The ratio between $A_{\mathrm{C}}$ and the attached current collector surface area $A_{\mathrm{CC}}$ sets our measure for the connectivity between cathode and current collector. Simulations of the effective conductivity in the active material phase are performed in the software GeoDict. ${ }^{?}$ The corresponding microstructure generator is described in detail in Sec. ??. Effective conductivities in through-plane direction $\sigma_{\mathrm{x}, \text { eff }}$ of the different virtual samples with varying contact ratio $\left(A_{\mathrm{C}} / A_{\mathrm{CC}}\right)$ are summarized in Fig. ??(b). As expected, we see in our simulations a decrease in the effective conductivity with decreasing contact area. In the extreme case of electrical contact at only $1 \%$ of the current collector surface, the effective conductivity decreases by almost $60 \%$ compared to our standard electrode. Although this virtual electrode certainly represents a worst-case scenario, it provides important insights into the qualitative effect of a limited contact between the electrode and current collector.

Particle Delamination The structural analysis confirms, that the calculated tortuosity of the solid electrolyte network $\left(\tau_{\mathrm{x}}=1.340\right)$ stays unchanged for all modified electrodes through the presented delamination algorithm in Sec. ??. Since we set the inactivity of the decontacted solid electrolyte selective towards the contacted active material, the lithium transport between inactive and active solid electrolyte stays unchanged. This particle delamination resembles the experimental conditions of a plain contact loss between NMC622 and $\beta$-LPS without material degradation. Nevertheless, the algorithm significantly reduces the active surface area in the cathode. Since larger fractions of particle surfaces are inactive 

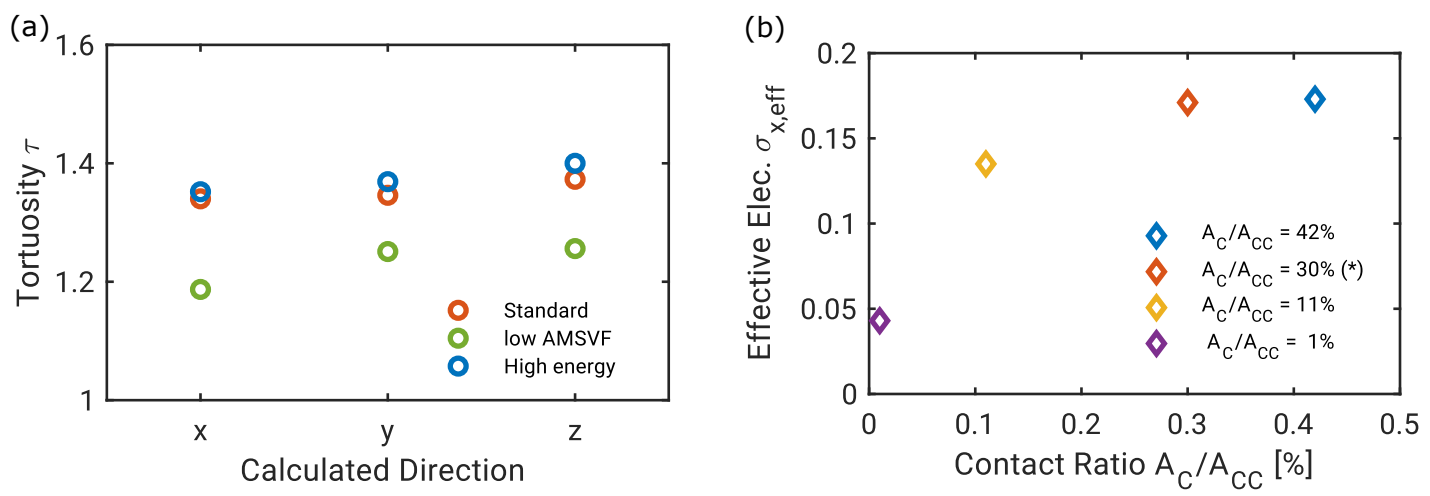

Figure 4: (a) Calculated tortuosities $\tau$ in x,y and z-direction for the different virtual electrodes: standard, low AMSVF, and high energy. (b) Effective electronic conductivities $\sigma_{x, e f f}$ in through-plane direction of virtual electrodes with varying contact area to the current collector. $*$ marks the standard electrode). Results are generated by the procedure given in Sec. ??.

towards the electrolyte, the effective Li-ion pathways have to extend in order to sustain the intercalation process. The different cases provide an estimate for the effect of incomplete wetting of the particles during electrode production, as well as the contact loss of the particles due to volume changes during cycling.

At the transition from the composite cathode to the solid electrolyte separator, we observe a weak change in the grey values towards a lighter color (cf. Fig. ??(b) yellow box). We interpret this as an artifact originating from a high external pressure. The softer separator with a shear modulus of $G=11.3 \mathrm{GPa}$ experiences a gradual compression through the stiffer NMC622 $(G=78 \mathrm{GPa})$ backbone. Nevertheless, the limited $1 \mu \mathrm{m}$ resolution of the CT measurement provides no insight on secondary phase formation of NMC622, contact loss of NMC622 particles with the electrolyte, or other interface related limitations. ? In our simulations, we assume in a first step ideally contacted interfaces. Therefore, we expect a better performance of our virtual cells compared to their counter-parts characterized in the experiments. 


\subsection{Performance Characterization}

In the following, the results for the electrochemical characterization for the fabricated standard and the high energy cell are discussed. The rate performance and cycling tests on the $\mathrm{Li} \mid \beta$-LPS|NMC622 pellet-type cells are performed as described in Sec. ??. Prior to the electrochemical tests, all cells were charged galvanostatically to $4.3 \mathrm{~V}$ vs $\mathrm{Li}^{+} / \mathrm{Li}$. Recent studies indicate that during the initial charging step, a solid electrolyte interphase (SEI) is forming on the uncoated active material, which acts as a protecting layer and minimizes the

cell degradation. ? In the subsequent rate tests the cells with $10 \mathrm{~mm}$ diameter were cycled between 2.6 and $4.3 \mathrm{~V}$ vs $\mathrm{Li}^{+} / \mathrm{Li}$ at $25^{\circ} \mathrm{C}$. The cells with $10 \mathrm{~mm}$ diameter were galvanostatically charged to $4.3 \mathrm{~V}$ and discharged to $2.6 \mathrm{~V}$ vs $\mathrm{Li}^{+} / \mathrm{Li}$ at $25^{\circ} \mathrm{C}$. The standard C-rate is 0.1C for prolonged cycling. Additionally, rate tests were performed at various higher rates. A detailed overview of the cell specifications and results is shown in the supporting information. For a detailed discussion of the results, we refer to the publication of the co-author. In this work, we focus on the validation of our simulated cell performance and select three exemplary discharge cycles for the standard cell at $0.1 \mathrm{C}, 0.25 \mathrm{C}$ and 0.5 as well as $0.1 \mathrm{C}$ for the high energy cell shown in Fig. ??(b). Furthermore, the consistency regarding the simulated interface processes and cell parametrization is verified via electrochemical impedance spectroscopy.

Capacity Retention A brief description of the typical electrochemical performance at low current is provided in the paragraph below. During the first charge step a high irreversible capacity is observed for all cell types (cf. Fig. S1 supporting information). The Coulomb efficiency for the first cycle of cell type 1 is $73 \%$. This first capacity loss indicates a stabilization at the electrode/solid electrolyte and active material/solid electrolyte interfaces (cf. Fig. S2 supporting information). The average Coulomb efficiency is $99.8 \%$, excluding the first cycle, indicating only small contributions of parasitic side reactions. For the high 
energy cell, comparable observations are made. The Coulomb efficiency of the first cycle is $71 \%$ and for the following cycles $99.2 \%$ (excluding the first cycle). The two cell setups showed a comparable capacity loss for the shown 100 cycles. The constant capacity loss during prolonged cycling is attributed to interfacial reactions at the cathode active material with the solid electrolyte and at the contact area of lithium metal and the solid electrolyte. A detailed XPS analysis for the degradation in the cathode composite is found in the article of Koerver et al. ${ }^{\text {? }}$ Additionally, the 5\% anisotropic particle reduction of NMC622 during lithiation and delithiation enhances the delamination of active particles? ${ }^{?}$ and amplifies the degradation through contact loss of some particles. A similar and even more pronounced mechano-chemical fading is observed in oxide-based ASSB systems where the mechanical fading is enhanced through the stiffer solid electrolyte.

Rate Limitation Finally, the performance of the standard and high energy cell are tested for various charge and discharge rates. The rate tests are performed by applying current densities of $0.178 \mathrm{~mA} / \mathrm{cm}^{2}, 0.445 \mathrm{~mA} / \mathrm{cm}^{2}, 0.89 \mathrm{~mA} / \mathrm{cm}^{2}$ and $1.78 \mathrm{~mA} / \mathrm{cm}^{2}$, which correspond to $0.1 \mathrm{C}, 0.25 \mathrm{C}, 0.5 \mathrm{C}$, and $1.0 \mathrm{C}$, respectively, based on a theoretical capacity of $200 \mathrm{mAh} / \mathrm{g}$ of the active material. For the standard cell, the specific capacities reduce from $128 \mathrm{mAh} / \mathrm{g}$ to $98 \mathrm{mAh} / \mathrm{g}, 54 \mathrm{mAh} / \mathrm{g}$ and $0 \mathrm{mAh} / \mathrm{g}$ (non cyclable) at $0.178 \mathrm{~mA} / \mathrm{cm}^{2}$, $0.445 \mathrm{~mA} / \mathrm{cm}^{2}, 0.89 \mathrm{~mA} / \mathrm{cm}^{2}$ and $1.78 \mathrm{~mA} / \mathrm{cm}^{2}$, respectively. Due to the lowering of accessible capacity, these current densities correspond to a true C-rate of $0.16 \mathrm{C}, 0.48 \mathrm{C}, 1.71 \mathrm{C}$, and n.d. For the high energy cell, the specific capacities reduce from $106 \mathrm{mAh} / \mathrm{g}$ to $78 \mathrm{mAh} / \mathrm{g}$, $46 \mathrm{mAh} / \mathrm{g}$ and $0 \mathrm{mAh} / \mathrm{g}$ at $0.356 \mathrm{~mA} / \mathrm{cm}^{2}, 0.89 \mathrm{~mA} / \mathrm{cm}^{2}, 1.78 \mathrm{~mA} / \mathrm{cm}^{2}$, and $3.56 \mathrm{~mA} / \mathrm{cm}^{2}$ respectively. Due to the lowering of accessible capacity, these correspond to a true C-rate of $0.19 \mathrm{C}, 0.66 \mathrm{C}, 2.22 \mathrm{C}$, n.d., respectively.

We experimentally observed two prominent mechanisms limiting the cycle stability and 
performance of sulfidic ASSB cells. Firstly, mechano-chemical degradation through particle swelling and secondly the secondary phase formation on anode and cathode side during long term cycling. Additionally, the strong rate limitation induced by the nonlinear internal resistance drastically diminishes the cell performance and capacity retention. Furthermore, short-circuiting of the cell through dendrite formation is another discussed mechanism limiting the cell lifetime. ${ }^{?}$ A description of the long-term cycling and failure mechanisms due to e.g. dendrite formation and secondary phase formation is not in the scope of this paper. Thus, we focus on the first couple of cycles, during which such long-term degradation mechanisms can be neglected.

\subsection{Model Validation}

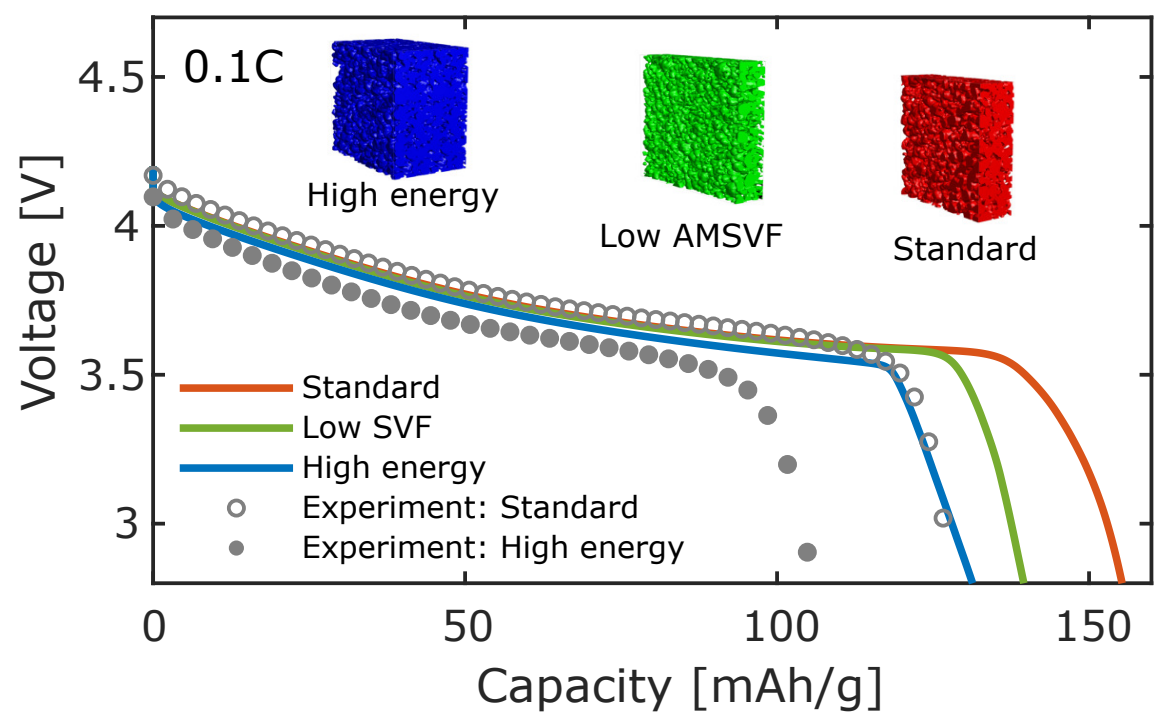

Figure 5: Comparison between experimental (gray symbols) and simulated 0.1C discharge curves for the virtual standard (solid red), low AMSVF (solid green), and high energy electrode (solid blue).

Cell Performance analysis at low currents In a first step, we simulate the discharge behavior and impedance spectra for the unmodified standard electrode in order to analyze the cell parameterization introduced in Sec. ??. Fig. ?? shows the cell voltage during a 0.1C discharge. Experimental data are represented by open symbols and simulations results 
are given by red solid lines. As expected, our discharge simulation with identical internal and external interfaces overestimates the experimental discharge capacity of $128 \mathrm{mAh} / \mathrm{g}$ by $\sim 20 \%$, but shows a generally good agreement with the cell voltage in the first half of the discharge.

As discussed in Sec. ??, some of the deviations are due to local fluctuations in the AMSVF, which are not fully captured in our reconstructed volume of the standard electrode. In order to provide a qualitative analysis of these fluctuations, we additionally performed discharge simulations of the virtual electrode with the lower active material content (low AMSVF). The results of the simulations are given by the solid green line in Fig. ??

The simulated discharge curve is in better qualitative agreement (deviation $\sim 8.5 \%$ ) with the experimental data. These two scenarios can be seen as limiting cases and on the macroscopical scale, one will only observe an average performance. Still, the results show that inhomogeneities in electrode loading locally affect the electrochemical behavior of the cell. The graph also contains the discharge curve of the virtual high energy cell at the same rate. In agreement with the experimental data, we observe a reduction of cell capacity, which indicates that an increase in electrode loading amplifies transport limitations and reduces active material utilization. A detailed discussion on limiting processes is given in the paragraph below.

Lithium distribution Fig. ?? shows the concentration distributions of $\mathrm{Li}$ in the active material during the $0.1 \mathrm{C}$ discharge at a cell voltage of $3.75 \mathrm{~V}$ (left), $3.3 \mathrm{~V}$ (middle) and $2.8 \mathrm{~V}$ (right) for the standard (top) and high energy configuration (bottom). After the evaluation of the concentration distributions, we identify two processes during lithiation that limit the active material utilization and cause a capacity loss. At the beginning of the discharge, lithiation predominantly occurs in the area close to the separator, which is due to the low ionic conductivity of the electrolyte. Later on, in the lithiation process, the electronic con- 
ductivity of the NMC622 starts to decrease due to the increasing lithium concentration. At this stage, the transport of electrons within the active material becomes the limiting process. As a consequence, we observe preferential intercalation close to the current collector. This is a self-limiting process, continuously increasing the Li concentration close to the current collector and, consequently, reducing the local conductivity.

Due to these two competing processes, we observe a sandwich like lithiation starting from the separator, which at lower SOCs shifts to a gradual lithiation close to the current collector. In our simulations, the decreasing electronic conductivity close to the current collector is the major source of the capacity loss. Our findings agree with a recent study by Bartsch et al. via operando X-Ray Diffraction, reporting the presence of two fractions of NMC622 with varying degree of lithiation during cycling. In our simulation, this effect is enhanced through the segregation of smaller particles close to the current collector (see Fig. ??(b)). Smaller particles tend to improved lithiation kinetics, which in turn leads to a faster decrease in the effective electronic conductivity. This inhomogeneous lithiation becomes clearly visible in thicker electrodes as demonstrated by the simulations of the virtual high energy cells with twice the cathode thickness of the standard cell.

Fig. ?? compares the simulated $0.1 \mathrm{C}$ discharge behavior of the high energy cell (solid red) to the standard setup (solid blue). Since the separator thickness for the high energy cell is halved $(210 \mu \mathrm{m})$ and the $0.1 \mathrm{C}$ discharge current is doubled $\left(0.356 \mathrm{~mA} / \mathrm{cm}^{2}\right)$ according to the increased specific capacity, we expect a comparable resistance loss at the separator and composite cathode as for the standard cell. Nevertheless, for the thicker electrode the cell voltage is generally lowered and we observe a capacity loss of $15 \%$. The cell voltage reduction for higher current densities is potentially enhanced through increasing dissipation losses at the lithium metal anode side.

The concentration distributions shown in Figs. ?? (c) and (f) highlight the effect of the competing ionic and electronic transport, resulting in the sandwich like lithiation explained above. The layer with low active material utilization in the center of the electrode is evident 
in Fig. ?? (f). Conductive additives could establish a better network for electronic conduction in order to mitigate inhomogeneous lithiation and capacity loss of ASSB composite cathodes. However, additional passive materials reduce the energy density of the cell and might be a source for further degradation mechanisms, which negatively affects the cycle life of the cell.

(a)

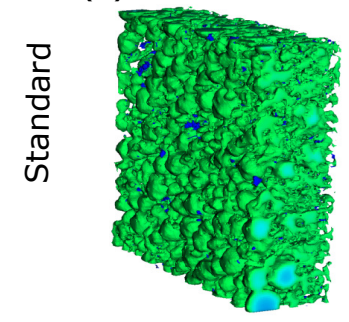

(d)

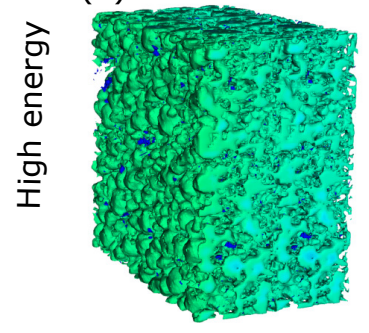

$\mathrm{OCP}=3.3 \mathrm{~V}$

(b)
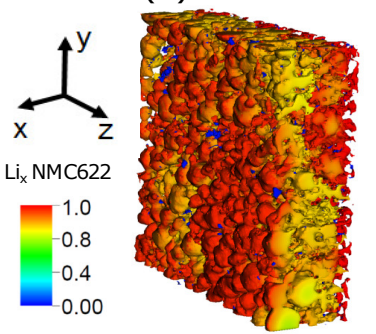

(e)

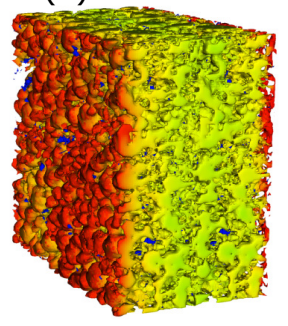

$\mathrm{OCP}=2.8 \mathrm{~V}$

(c)

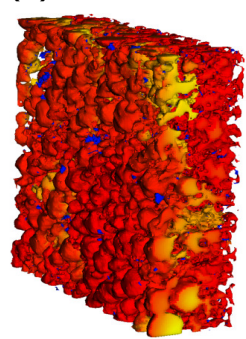

(f)

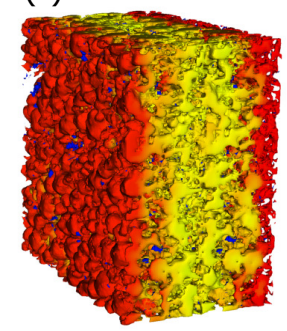

Figure 6: (a),(b) and (c) lithium distribution in the active material of the standard composite cathode $(40 \mu \mathrm{m})$ at cell voltages of $3.75 \mathrm{~V}, 3.3 \mathrm{~V}$ and $2.8 \mathrm{~V}$ during a $0.1 \mathrm{C}$ discharge. (d), (e), and (f) show concentration distributions in the high energy cathode $(80 \mu \mathrm{m})$ under the same conditions.

Impedance analysis In a next step, we compare the simulated impedance response with the experimental data in order to deduce information on the characteristic time scale of different processes. Fig. ??(a) shows measured (open symbols) and simulated (line) impedance spectra of the standard cell in the charged state $(\mathrm{OCP}=4.2 \mathrm{~V})$. The high-frequency response belongs to the grain and grain boundary transport in the polycrystalline solid electrolyte separator (green). The stretched out semi-circle in the mid-frequency range is assigned to the charge transfer at the Li metal anode (blue) and NMC622 cathode (red), respectively. At 
even lower frequencies one can see the onset of the contribution of Li diffusion in the active material, which is not fully resolved in the measurements.

The simulated impedance response qualitatively reproduces the spectra at lower frequencies. In our simulations we do not include the effect of grains and grain boundaries on transport in the solid electrolyte. Thus, the semi-circle at high frequencies is not resolved in our simulations. Instead the overall solid electrolyte bulk resistance $R_{\text {Elyte }}$ given by the intersection of the imaginary part with the real axis includes bulk and grain boundary transport. In the measurements we use the intersection with the real axis at around $361 \Omega \mathrm{cm}^{2}$ as an indicator for $R_{\text {Elyte }}$. Moreover, the simulated impedance spectra are in favorable agreement with the experimental data, reproducing the stretched out semi-circle at intermediate frequencies as well as the solid electrolyte bulk resistance. Based on our analysis of characteristic frequencies of the charge transfer reactions, we can assign the semi-circle around $31 \mathrm{kHz}$ to the $\mathrm{Li}$ electrode and the semi-circle around $2.5 \mathrm{kHz}$ respectively to the intercalation reaction at the cathode. This interpretation is in line with the impedance measurements on the symmetric Li cells. This indicates that the kinetic parameters extracted from the measurements as described in Sec. ?? provide reasonable estimates for the anode and cathode charge transfer reactions.

Performance analysis at high currents Finally, we want to discuss the performance of $\beta$-LPS based ASSBs at high discharge currents. Experimental data is shown in Fig. ??(b) and we observe a significant capacity decay at high C-rates of up to $60 \%$. Fig. ??(b) additionally includes simulated discharge curves of the standard electrode setup (solid red $=$ $0.1 \mathrm{C}$, dashed red $=0.25 \mathrm{C}$, dotted red $=0.5 \mathrm{C}$ ). The simulations overestimate the discharge capacity compared to the experiments. Moreover, the overpotentials in the simulations are significantly lower than in the experimental data. Since our simulations are able to reproduce the impedance spectra at the beginning of discharge, we conclude that there need 
(a)

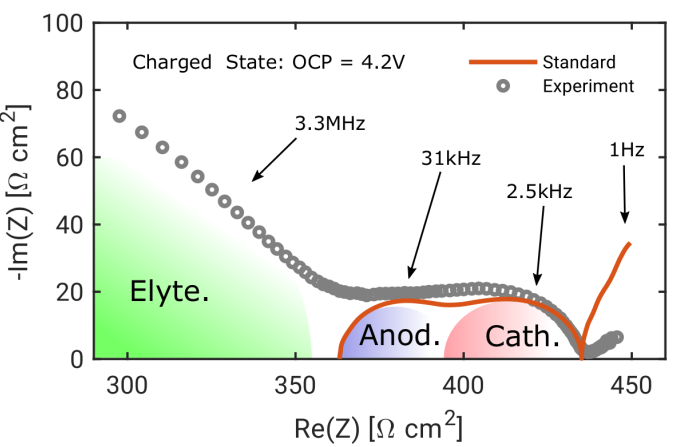

(b)

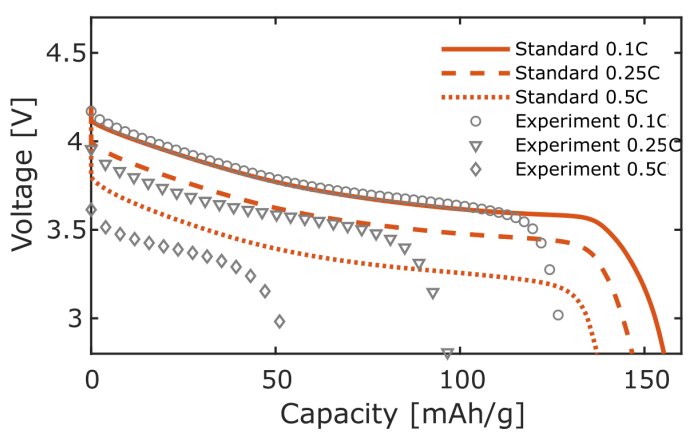

Figure 7: (a) Simulation (line) and measured (symbol) impedance spectra for a charged cell $(\mathrm{OCP}=4.2 \mathrm{~V})$ with the standard electrode. (b) Results of the C-rate test measured (symbols) and simulated (lines) on the standard electrode.

to be additional processes, which become apparent only during the dynamic discharge.

Possible candidates discussed in literature are (I) space charge layers at the electrode to electrolyte interface? ? as well as (II) in the polycrystalline solid electrolyte itself ${ }^{\text {? }}$ and (III) morphological changes in the electrode due to the shrinking and swelling of particles such as contact loss and delamination. ? (I) and (II) are important aspects of the research within our groups but are not in the focus of this work. In this article, we want to address the effect of morphological changes during cycling of the composite electrodes. As explained above, we do not include mechanical stress effects in our microstructure-resolved simulations. Instead, we compare different defined geometrical scenarios described in Sec. ??. The results of this performance analysis at high currents are presented in the next section. 


\subsection{Microstructural Modifications}

The simulations predict specific capacities, which exceed the experimental data, especially, at high currents. The deviation can not be solely attributed to local fluctuations in the AMSVF identified in the reconstructed virtual electrodes in Sec. ??. This indicates that further effects either in the constitutive equations itself or the corresponding microstructure model need to be taken into account. In this article, we focus on the influence of morphological changes of the electrode microstructure such as a reduced contact of the electrode layer to the current collector (cf. Sec. ??) or contact loss of active material to the solid electrolyte network due to volume changes (cf. Sec. ??). The effect of these scenarios on structural properties is analyzed in Sec. ?? and the results imply that the modifications influence the cell voltage and capacity. In the following paragraphs, electrochemical simulation results for virtual electrodes with the structural modifications introduced above will be discussed in more detail.

We want to emphasize that the modifications of the microstructure are based on experimental observations by various groups? ? ? and are partly based on our structural analysis. Therefore, the modified structures represent virtual scenarios, which could be present in a real electrode.

\subsubsection{Cathode to Current Collector Interface}

In our simulations of virtual cells with the standard composite cathode described in Sec. ??

we see that agglomerates of smaller particles at the current collector lithiate more rapidly than larger particles in this area. Since the electronic conductivity of lithiated NMC622 reduces by orders of magnitude, ${ }^{?}$ ? preferentially lithiated particles reduce the effective electronic conductivity of the electrode close to the current collector. On the other hand, very large particles with the same volume fraction tend to have only few contact points with the current collector. Locally, the current focuses on only a few bottlenecks that are pref- 


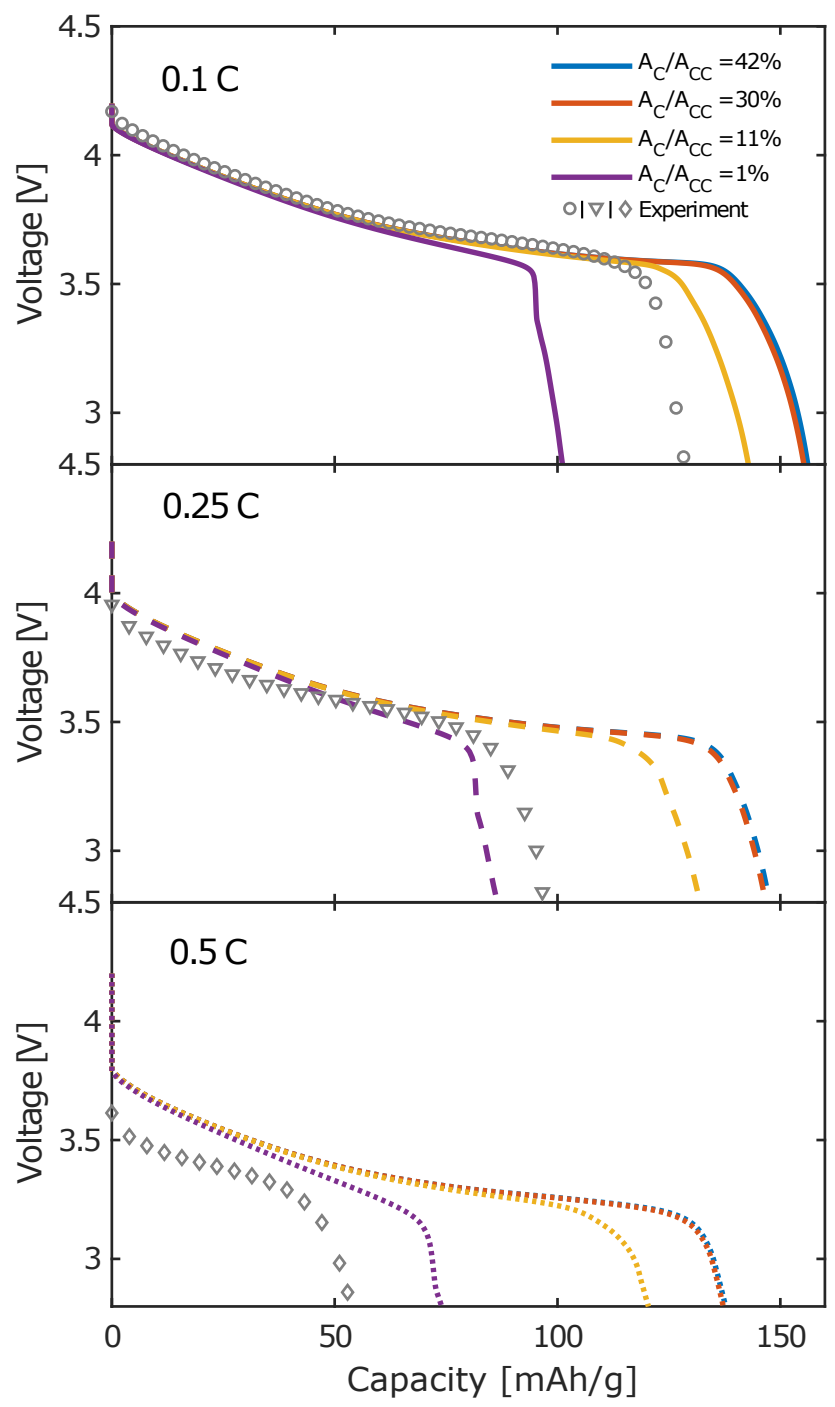

Figure 8: Discharge capacity for different C-rates under the influence of contact loss between cathode active material and current collector. Experimental data is given by open symbols.

erentially lithiated. By reducing the contact area in our simulations, we expect preferential lithiation of the active material close to the current collector, which amplifies the loss of electronic conductivity in these distinct areas (see Fig. ??).

Discharge curves Fig. ?? shows the influence of contact area between cathode $\left(A_{\mathrm{C}}\right)$ and current collector $\left(A_{\mathrm{CC}}\right)$ on the discharge curves at different C-rates. In the graph, the contact area is normalized to the current collector area. By decreasing the connectivity to 
the current collector, the specific discharge capacity of the virtual cells reduces significantly. Between the virtual electrodes with $42 \%$ (blue line) and $30 \%$ (red line) normalized area, we observe only a marginal loss in capacity in the range of $2 \%$. We conclude that even though the contact area reduces by $12 \%$, electronic pathways are still sufficient to provide a high cathode utilization. For the case of $11 \%$ normalized contact area represented by the yellow line in Fig. ??, the capacity reduces by $9 \%$ compared to the virtual standard electrode with $30 \%$ normalized contact area. Still, the specific capacity at high currents is significantly larger than in the experimental data. However, in the extreme case of a contact area of only $1 \%$ (violet) of the current collector area we see a drop in the specific capacity even for the 0.1C discharge, which is enhanced at high currents. Since we distinguish only between "no contact" and "perfect contact" (no additional contact resistance), the reality is expected to exhibit additional resistances due to imperfect contact.

Lithium distribution Fig. ?? shows the Li concentration (left column) and local current density (right column) for the virtual electrodes with $30 \%$ (top) and $11 \%$ (bottom) normalized contact area during a $0.1 \mathrm{C}$ discharge at a cell voltage of $3.6 \mathrm{~V}$. In order to visualize the concentration distribution at the surface towards the current collector, the virtual electrodes are rotated. The virtual electrode with $30 \%$ normalized contact area shows a relatively homogeneous active material utilization (cf. Fig. ??(a)) at the current collector interface as well as over the whole composite cathode. In contrast, the virtual electrode with $11 \%$ normalized contact area in Fig. ??(c) exhibits very localized lithiation of only a few particles in contact with the current collector in the bottom part. The preferential lithiation of these particles reduces their electronic conductivity and, thus, the overall contact with the current collector. Hence, the preferential path for electrons is essentially blocked, which causes a capacity loss during discharge. Since the electronic degradation process is self-accelerating, increasing the discharge rate amplifies the blocking behavior. 
Conducting network Figs. ??(b) and (d) present the local current density of the two virtual electrodes at the same time step. In these graphs, the selective lithiation due to the reduced number of percolating pathways in the electrode with the lower contact area becomes evident. The current density distribution of the virtual electrode with the $11 \%$ normalized contact area reflects the concentration distribution in Fig. ??(c). Only at a few remaining particles a current can be observed, which is, however, strongly enhanced compared to the more homogeneous case with larger contact area (see Fig. ??(c)). In this case, the additional electronic pathways improve the current distribution and, thus, the active material utilization.

This result highlights, that above all, intimate electronic contact between the electrode layer and the current collector needs to be ensured. Similar findings are reported for conventional lithium-ion batteries with a carbon primed current collector. ${ }^{?}$ The contact loss to the current collector can be further amplified by the volume change of NMC622 particles during delithiation, leading to a reduction of electronic pathways and, possibly, inactive areas within the cathode. In order to prevent these degradation phenomena due to localized lithiation of the active material, crucial parameters are the particle size and a homogeneous particle distribution within the composite cathode. Previous simulation studies on the electrode structure by Bielefeld et al. ${ }^{?}$ demonstrated the dependency of the potential cell performance on the electronic and ionic percolating pathways, particle sizes and electrode porosity. For instance, the particle size distribution is strongly influenced through the processing routine e.g. the milling time and the batch size. ? Hence, these parameters determine the effective electronic conductivity and, therefore, influence the internal as well as the external interfaces of the cathode. Besides the adjustments of production parameters, the introduction of conductive additives, which form a percolating conductive network is one approach to improve 
the cathode performance.

Although our simulations provide evidence that imperfect contact between the current collector and the electrode layer reduces the capacity of the cell, we do not observe a reduction of cell voltage, which is very prominent in the experimental data. Even if the normalized contact area is only $1 \%$, we see only a marginal reduction of the cell voltage. Therefore, we additionally investigate the effect of contact loss between active material particles and the solid electrolyte in the next paragraph.

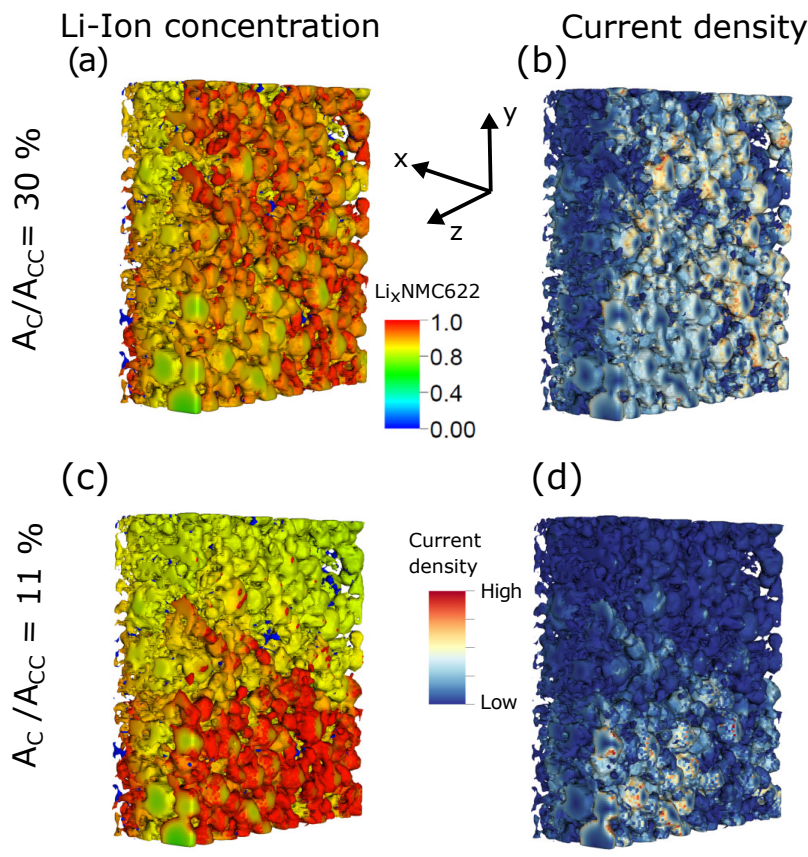

Figure 9: Comparison of the Li-ion concentration and current density distribution between $30 \%$ (standard electrode) and $11 \%$ (Li-concentration $=(\mathrm{a}),(\mathrm{c})$; current density $=(\mathrm{b}),(\mathrm{d})$ ) relative contact area at a cell voltage of $3.6 \mathrm{~V}$. Rotated microstructure: view on cathode from the current collector side.

\subsubsection{Particle Delamination}

The performance analysis in Sec. ?? indicates that during the first charge and discharge $27 \%$ of the theoretical capacity is lost due to contact loss of active material particles (particle 


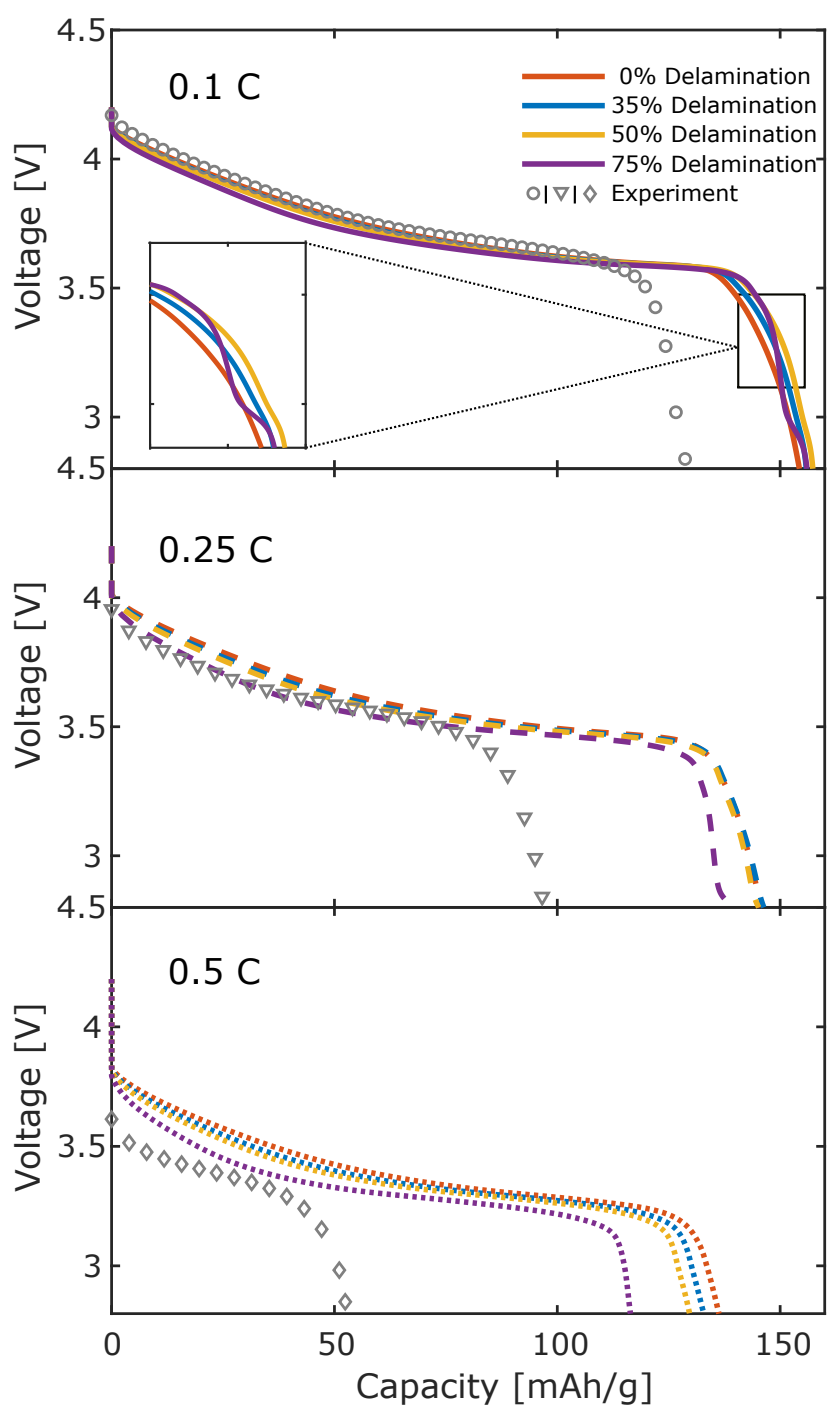

Figure 10: Discharge capacity for different ratios of solid electrolyte delamination on the cathode side (solid lines). The corresponding experimental measurements are given by open symbols. (a) Zoom into the $0.1 \mathrm{C}$ discharge close to the cutoff voltage.

delamination). ? The linear capacity fade during cycling (cf. Fig. S2 supporting information) suggests that the contact loss of NMC622 particles due to volume changes does not stop after the initial charge process. Other effects, like the formation of secondary phases, additionally contribute to the capacity loss. Although our model does not simulate changes in electrode microstructure during cycling, we are still able to investigate the effect of different microstructural modifications, which mimic the effects induced by the stress inside the ASSB or imperfect contact during electrode preparation. The corresponding microstructure 
generator, which emulates the delamination of the solid electrolyte from the active particle surface is described in Sec. ??. In this paragraph, we present results of our electrochemical simulations.

Discharge curves First, we analyze the effect of a loss of particle contact on the specific capacity during discharge. Fig. ?? shows the discharge capacity for three different degrees of delamination at varying C-rates. The values indicating the degree of delamination in Fig. ?? are given by the ratio of the passive particle surface and the total active particle surface. Generally, we see that with increasing delamination, the overpotential in the discharge curves also increases. At low C-rates the difference between the virtual electrodes is minor. However, at high currents, the effect is relatively prominent. Longer transport pathways due to the reduced active surface area start to have a visible effect on the cell voltage at $0.25 \mathrm{C}$ discharge current.

The larger the contact loss of the active particles to the solid electrolyte, the more pronounced is the resulting overpotential. This effect is most prominent at a $0.5 \mathrm{C}$ discharge rate. For the highest degree of delamination with a reduction of active surface area by $75 \%$, we see a potential drop, which is similar to the one observed experimentally. Nevertheless, for the $0.1 \mathrm{C}$ and $0.25 \mathrm{C}$ rate, the specific discharge capacity is still close to the capacity of the unmodified reference electrode. At the highest discharge current, corresponding to a $0.5 \mathrm{C}$ rate, the specific capacity is around $17 \%$ less compared to the unmodified virtual electrode. This confirms that the network for Li transport is not destroyed but the electrolyte transport is significantly influenced by the microstructure modifications.

Extended discharge For the simulated discharge at $0.1 \mathrm{C}$ we observe an additional effect induced by the electrolyte decontacting, which is the increase in discharge capacity for growing delamination ratio (cf. Fig. ??(a)). This effect climaxes for the electrode with 

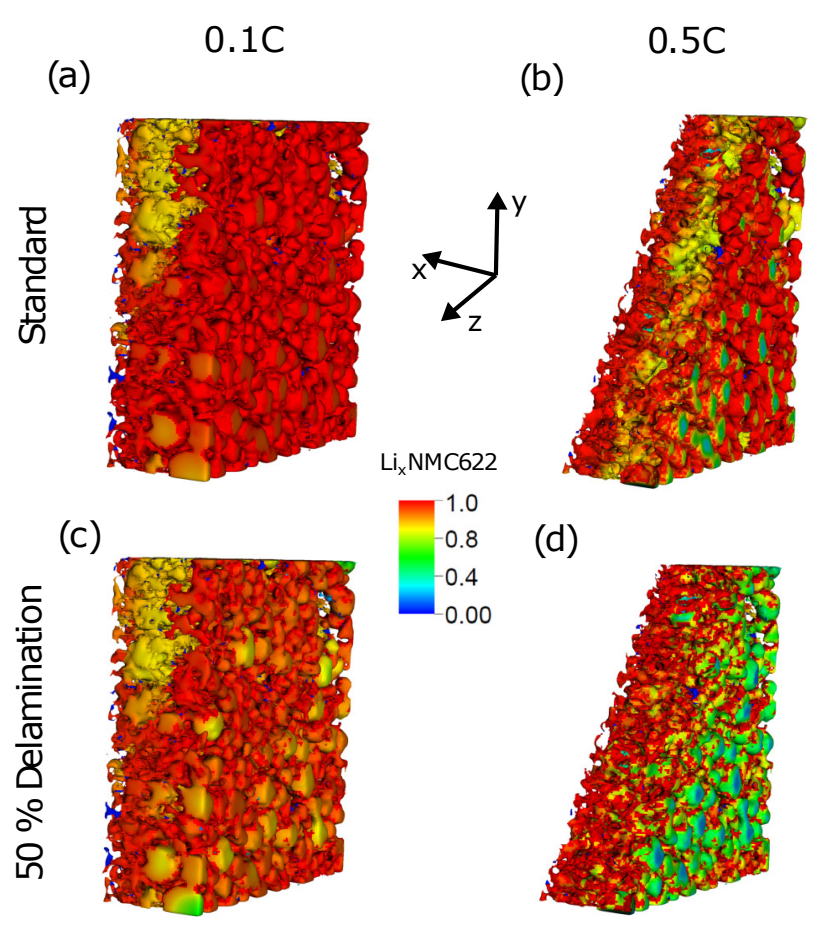

Figure 11: Concentration distribution for virtual electrodes with unmodified microstructure (top) and $50 \%$ delamination (bottom) close to the lower cut-off voltage $(3.0 \mathrm{~V})$ of a $0.1 \mathrm{C}$ (left column) and $0.5 \mathrm{C}$ (right column) discharge simulation. Rotated microstructure: view on cathode from the current collector side. The sliced view for the $0.5 \mathrm{C}$ discharge shows the concentration gradient within the active material.

$75 \%$ inactive surface area, in a two-staged lithiation process close to the cutoff voltage. This staged lithiation originates through the increased lithium transport pathways in the modified electrode. The intercalation process is amplified at the remaining active material surfaces. Therefore, the areas close to the separator lithiate more rapidly and staggering the transport to the current collector. This stalling effect enables a higher cathode utilization since an early reach of the cutoff voltage through the electronic degradation of the active material is delayed. By comparison of the lithium concentration distribution shown in Fig. ??, this effect becomes evident.

Lithium distribution Fig. ?? shows the Li concentration in NMC622 for the unmodified standard electrode (top) and the case with $50 \%$ loss of active surface area (bottom) close to a 
cell voltage of $3.0 \mathrm{~V}$. The standard electrode in Fig. ??(a) displays a homogeneously lithiated cathode surface towards the current collector. The sandwich-like lithiation discussed in Sec. ?? creates the electronically blocking interface that prevents a further cathode utilization. In contrast, the electrode with $50 \%$ delamination (Fig. ??(c)) shows align inhomogeneous lithiation at the current collector interface. The remaining sufficiently conducting active material particles provide electronic pathways to extend the intercalation process and raise the discharge capacity.

For the higher $0.5 \mathrm{C}$ discharge rate, the effect of the reduced active surface area is even more visible (Fig. ??(d)) and the amplified lithiation close to the separator becomes more prominent. The restricted access of Li from only a fraction of the particle surface creates concentration gradients within the particle. Moreover, the gradual sandwich-like lithiation observed for the standard electrode in Fig. ??(b) is diminished by the microstructure modifications, indicating that the solid electrolyte transport is the limiting mechanism. Furthermore, for higher C-rates, the amplified lithiation close to the separator superimposes with the faster degrading electronic conductivity in the active material, leading to increased losses in the discharge capacity.

Impedance analysis For a better understanding of the effects of particle delamination, we also perform impedance simulations. The simulation results of the virtual electrodes with different degrees of contact loss are shown in Fig. ??. Additionally, we include the corresponding impedance measurements in the first and 11th cycle. For a better comparison, we normalized the simulated and experimental impedance data by subtracting their corresponding separator bulk resistance $R_{\text {Elyte }}$.

The growth in bulk solid electrolyte resistance during cycling is much more pronounced in the measurements, increasing by $\sim 10 \%$ from $R_{\text {Elyte }}=361 \Omega \mathrm{cm}^{2}$ to $R_{\text {Elyte }}=400 \Omega \mathrm{cm}^{2}$. This indicates an additional degradation mechanism such as electrolyte decomposition or 
secondary phase formation, which we do not take into account in our model. The reduction of the active material surface increases the interface resistance, which is confirmed by the impedance simulations. In our simulations, we clearly see an increase of the second semicircle corresponding to the cathode charge transfer reaction. Since the contact loss of the solid electrolyte with active particles is only introduced at the cathode, the interface resistance at the anode does practically not change.

Interestingly, the impedance simulations of the virtual electrode with an active surface area reduced by $75 \%$ show, although shifted by the bulk resistance, qualitatively the same impedance spectra like the measurements after the 11th cycle (Fig. ??). This implies a

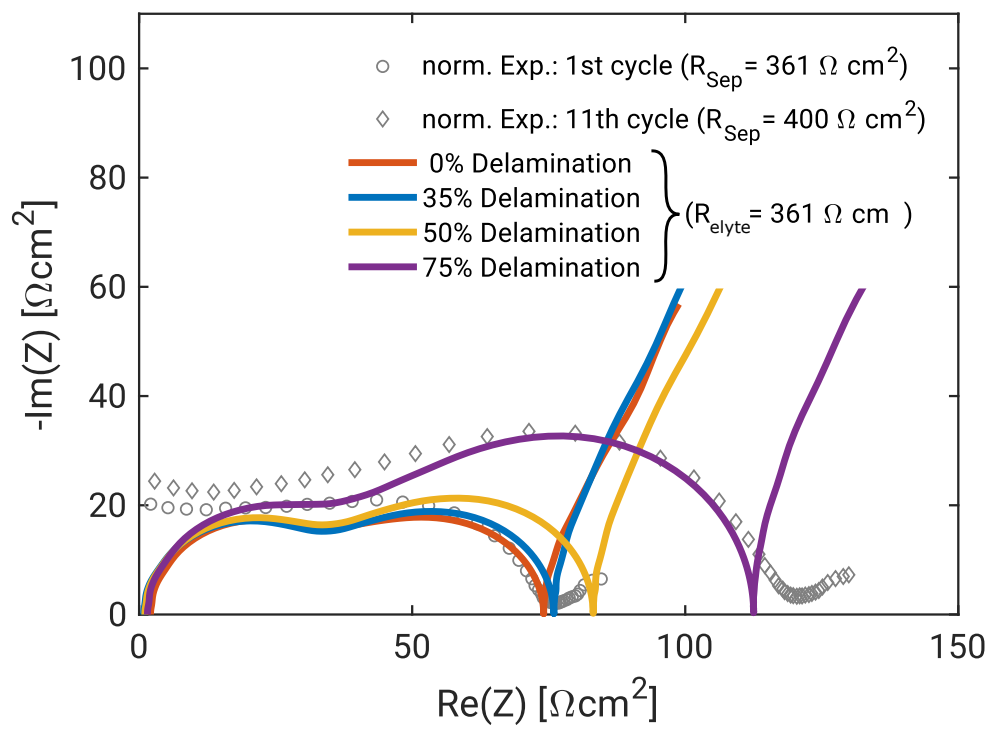

Figure 12: Simulated impedance for each delamination scenario (solid lines) and experimental impedance spectra after the first and 11th cycle. All data is shifted by the corresponding solid electrolyte bulk resistance $R_{\text {Elyte }}$ as indicated in the legend.

degradation of the cathode interface during cycling. However, our simulations currently do not allow us to distinguish between different degradation processes since the formation of passivating phases has a similar influence on the impedance as the loss of particle contact area investigated in this paper. On the other hand, the results suggest that it is not totally clear whether the increase in interface resistance after cycling can be entirely attributed to 
secondary phase formation. ${ }^{?}$ Therefore, we assume that the two mechanisms, and maybe others, superimpose in the experimental impedance spectra. Complementary measurement techniques are needed to investigate this issue.

In order to prevent a rapid particle delamination during cycling, typically an external pressure is applied to the cells to limit the effect of volume changes. Various studies on oxideand thiophosphate-based cells have shown that this approach is beneficial for the interface resistance and the cycle life for both anode and cathode. ? ?

An alternative approach to improve the active material contact and reduce the delamination during cycling could be achieved by combining solid electrolytes with polymers or ionic liquids. These softer hybrid electrolytes may enable a more intimate contact even with cathode materials that show volume changes during cycling. ? ? ? Our results show that delamination is a major interface parameter influencing the overpotential and cathode utilization. Therefore, this material aspect has to be addressed in the future cell design in order to enable ASSBs with nickel rich oxides.

\section{Conclusion}

In this paper, we present a study combining measurements and simulations of thiophosphatebased all-solid-state batteries. The 3D microstructure-resolved simulations are run on reconstructed electrodes obtained by CT measurements of $\beta$-LPS/NMC622 composite cathodes. This modeling concept inherently accounts for tortuosity effects and structural inhomogeneities. The cell computational results are validated by experimental data using both discharge measurements and electrochemical impedance spectra.

Based on this approach, we are able to identify two competing mechanisms. Primarily, capacity losses are caused by the decreasing electronic conductivity of the active material 
during lithiation. This self-accelerated electronic blocking of the cathode, especially at higher rates, detrimentally lowers the cathode utilization close to the current collector. Secondly, the relatively low ionic conductivity of the solid electrolyte promotes the intercalation close to the separator.

The competition of both mechanisms leads to an inhomogeneous and imperfect utilization of the active material, resulting in an interesting sandwich-like lithiation of the active material, especially pronounced in thicker electrodes with higher energy density. These inherent material properties restrict cell performance and are potentially enhanced by morphological inhomogeneities.

In this article, we investigate the effect of two morphological changes, which possibly occur during the operation of the cell: (I) a reduced contact of the electrode layer to the current collector and (II) the delamination of the solid electrolyte from the active particle surface.

(I) A small contact area between the active material and current collector reduces the specific capacity at high currents. Enhanced local currents cause inhomogeneous lithiation close to the current collector, which triggers a self-accelerated local reduction of electronic conductivity. This effect is able to reduce the specific capacity of the cell as it destroys the conductive network through the particles. In this context, the particle size and distribution are critical for cell performance. Moreover, simulations suggest that improved electronic contact with the current collector by coated current collectors as well as additional conductive additives in the bulk of the electrode might provide ways to improve high rate performance.

(II) The study on particle delamination shows that large overpotentials at high discharge rates might be coupled to the reduced active surface caused by volume changes of the active material within the composite cathode during cycling. Still, this effect does not seem to provide an explanation for the loss in specific capacity at high currents. However, in combination with other phenomena such as the formation of space charge layers in the solid electrolyte, further studies are needed to unravel the contributions 
to charge transfer kinetics.

Our simulations demonstrate, that both the internal and external interfaces of the composite cathode influence cell performance. Additional methodological developments, both theoretical and experimental, are needed to get a clear picture of the contribution of different processes at the interface of the solid electrolyte with the active material. This will be key to improve both the performance and cycle life of ASSBs.

\section{Acknowledgement}

Financial support from the Federal Ministry of Education and Research (BMBF) within the FELIZIA project (03XP0026F, 03XP0026J) and the FestBatt project (03XP0174C, 03XP0177A) is gratefully acknowledged. The authors acknowledge support with computational resources provided by the state of Baden-Württemberg through bwHPC (bwForCluster JUSTUS). The use of the X-ray microscope facilities funded by EPSRC Grant EP/M02833X/1 "University of Oxford: experimental equipment upgrade" is gratefully acknowledged. We also thank Lea Sophie Kremer for providing us the experimental GITT measurements on the cathode material NMC622.

\section{Supporting Information Available}

The following files are available free of charge.

- Supporting information: additional experimental information on dis/charge behavior, cell assembly, and impedance data; symmetric cell measurement on $\beta$-LPS ; CT image analysis; model parametrization equations 


\section{Graphical TOC Entry}

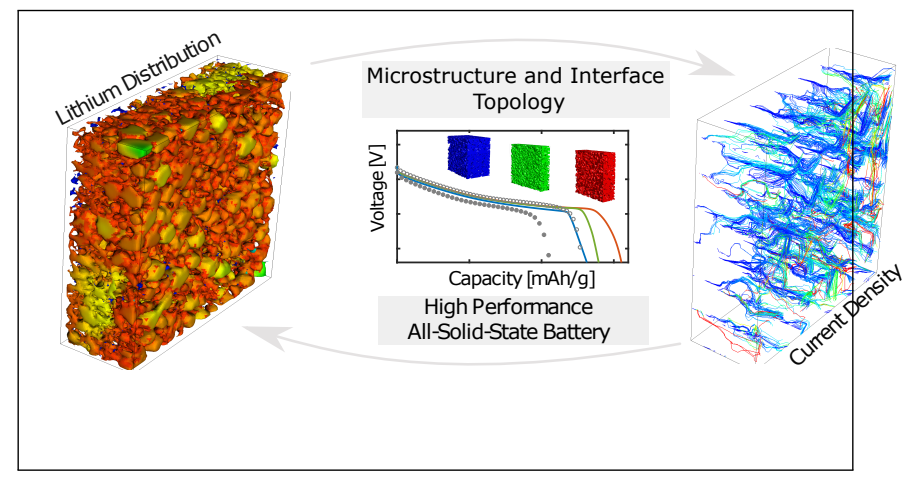

